67. Tagung der Vereinigung Norddeutscher Augenärzte 16.-17. Juni 2017 Westerland, Deutschland

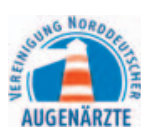

www.norddeutsche-augenaerzte.de $\rightarrow$ Nächste Tagung

\section{Jahrestagung der Retinologischen} Gesellschaft

23.-24. Juni 2017

Stuttgart, Deutschland

rg2017.congresse.de

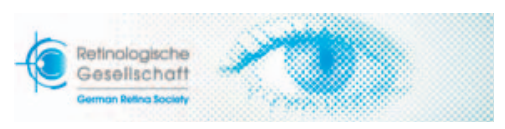

20. Tagung der BielschowskyGesellschaft für Schielforschung und Neuroophthalmologie 30. Juni-01. Juli 2017 Gießen, Deutschland www.bielschowsky.de

BIELSCHOWSKY
GESELLSCHAFT -

Sommertagung der BerlinBrandenburgischen Augenärztlichen Gesellschaft (BBAG) e.V.

8. Juli 2017

Berlin, Deutschland

www.bbag-augen.de $\rightarrow$ Veranstaltungen
25. Jahrestagung der Gesellschaft der Augenärzte Sachsen-Anhalts und Thüringens (SATh)

1.-2. September 2017

Jena, Deutschland

www.sath-augen.de

\section{SATh}

DOG 2017 - Deutsche Augenheilkunde international

28. September-1. Oktober 2017

Berlin, Deutschland

dog2017.dog-kongress.de

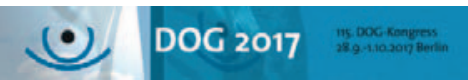

MAI

123e Congrès de la Société Française d'Ophtalmologie

6.-9. Mai 2017

Paris, Frankreich

www.sfo.asso.fr $\rightarrow$ Congrès $\rightarrow$

Congrès SFO 2017

30. Internationaler Kongress der Deutschen Ophthalmochirurgen (DOC) 11.-13. Mai 2017

Nürnberg, Deutschland

www.doc-nuernberg.de

Münsteraner Fortbildung für Augenärzte 17. Mai 2017

Münster, Deutschland

klinikum.uni-muenster.de $\rightarrow$ Kliniken \&

Institute $\rightarrow$ Augenheilkunde $\rightarrow$ Veranstaltungen

8. Dessauer Ophthalmologisches

Wochenende

19.-20. Mai 2017

Dessau, Deutschland

www.dessau-augen.de

58. Jahrestagung der Österreichischen Ophthalmologischen Gesellschaft (ÖOG)

25.-27. Mai 2017

Villach, Österreich

www.augen.at $\rightarrow$ Veranstaltungen
JUNI

European Society of Ophthalmology (SOE) 10.-13. Juni 2017

Barcelona, Spanien

soevision.org/congress

\section{Siebter St. Galler Ophtag}

22. Juni 2017

St. Gallen, Schweiz

www.kssg.ch/augenklinik $\rightarrow$ Lehre \&

Forschung $\rightarrow$ Weiter- und Fortbildung

JULI

\section{Augenärzteabend in Freiburg}

14. Juli 2017

Freiburg, Deutschland

www.uniklinik-freiburg.de/augenklinik $\rightarrow$

Für Ärzte $\rightarrow$ Augenärzteabende

Würzburger Glaukom-Tag mit LeydheckerHarms Ehrenvorlesung

15. Juli 2017

Würzburg, Deutschland

www.augenklinik.ukw.de $\rightarrow$ Veranstaltungen
AUGUST

110. Kongress der Schweizerischen Ophthalmologischen Gesellschaft (SOG) 30. August-1. September 2017

Locarno, Schweiz

www.sogcongress.ch

\section{SEPTEMBER}

16th EURETINA Congress -

European Society of Retina Specialists

07.-10. September 2017

Barcelona, Spanien

www.euretina.org $\rightarrow$ Barcelona 2017

20th Congress of the European Association for Vision and Eye Research (EVER)

27.-30. September 2017

Nizza, Frankreich

www.ever.be

OKTOBER

XXXV Congress of the European Society of Cataract and Refractive Surgeons (ESCRS)

7.-11. Oktober 2017

Lissabon, Portugal

www.escrs.org

\title{
KARGER
}

(C) 2017 S. Karger GmbH, Freiburg 